Globale Finanzmärkte, Nachhaltigkeit und der Beitrag "grüner" Investoren

\section{Zivilisierung ist Vorbedingung}

\author{
Nachhaltige Investitionen sind zum Teil auf die Finanzierung über globale Kapi- \\ talmärkte angewiesen. Die Nevordnung und Liberalisierung der Kapitalmärkte \\ seit den siebziger Jahren haben aber ein internationales Finanzsystem geschaf- \\ fen, das die Finanzierung von Investments mit ökologischen oder ethischen \\ Zielen erschwert.
}

$\mathrm{E}$ Von Kurt Hübner s stimmt: Geld kann man nicht essen! Richtig ist aber auch: Kapitalistische, arbeitsteilige Gesellschaften funktionieren ohne Geld nicht. Moderne Volkswirtschaften sind anonyme Tauschökonomien, in denen Akteure sich des Mediums Geld bedienen, um Transaktionen zu bewerkstelligen. Ohne monetären Standard könnten Transaktionen gar nicht oder nicht so effizient abgewickelt werden wie es augenblicklich geschieht. In einer profitgetriebenen Ökonomie bestimmen die Geld- und Kapitalströme letztlich über die Menge der produzierten und nutzbaren Güter und Dienstleistungen. Geld ist in diesem Zusammenhang mehr als Bargeld oder ein einfacher Bankkredit. Geld umfasst in einer modernen kapitalistischen Ökonomie alle Formen finanzwirtschaftlicher Titel, die von ökonomischen Akteuren mit unterschiedlichsten Motiven zur Bewerkstelligung ihrer auf Gegenwart und Zukunft bezogenen Kalküle verwendet werden. Neben dieser zeitlichen Dimension weisen Finanzmarkttitel auch eine räumliche Dimension auf: Geld und finanzwirtschaftliche Aktiva werden benötigt, um grenzüberschreitende ökonomische Transaktionen durchführen zu können. Dazu zählt die Abwicklung der Importe und Exporte von Gütern und Dienstleistungen genauso wie die Durchführung aller Arten von Direkt- und Portfolioinvestitionen.

\section{Zukunftsmärkte gewinnen an Relevanz}

Seit der Auflösung der Nachkriegs-Weltwirtschaftsordnung in den 1970er-Jahren ist ein enormes Wachstum grenzüberschreitender finanzwirtschaftlicher Transaktionen zu beobachten. Dies hat teilweise damit zu tun, dass der grenzüberschreitende Waren- und Dienstleistungshandel stetig zunimmt und immer mehr Unternehmen ihre Produktion internationalisieren, was zu einer Zunahme der handelsindu- dann bis 2000 auf 1300 Milliarden US Dollar pro Tag zu steigen. Mit dem Platzen der Börsenblase und der zunehmenden Unsicherheit über die weltwirtschaftlichen Entwicklungen ging der Future-Umsatz zwar bis 2002 wieder leicht zurïck, bewegt sich seit dem aber immer noch auf einem enorm hohen Niveau.

\section{Dynamik der Kapitalmärkte}

Die seit den achtziger Jahren auf der ganzen Welt verfolgte Liberalisierungspolitik hat aus den internationalen Geld- und Kapitalmärkten ein finanzwirtschaftliches Regime mit Zügen relativer Verselbständigung gemacht. Durch eine Vielzahl von Finanzmarktinnovationen und der weitgehenden Abschaffung von Mobilitätsrestriktionen für Kapital vorangetrieben, stiegen die Umsätze der internationalen Finanzmärkte sehr viel schneller als etwa das Volumen der grenzüberschreitenden Waren- und Dienstleistungstransaktionen oder gar das Volumen des Weltsozialprodukts. Nur das Tempo der ausländischen Direktinvestitionen konnte annähernd mit dem Wachstum der Finanzmärkte mithalten (siehe Tabelle 1).

Das Wachstum der Finanzmärkte ist allerdings nicht automatisch mit einer Verselbständigung der Finanzmärkte jenseits der tatsächlichen Wertschöpfung gleichzusetzen (1). Finanzmärkte haben die Aufgabe, zukünftige Investitionen zu finanzieren, Wertaufbewahrungsmedien bereit zu stellen und intertemporale Kontraktfunktionen auszuüben. Durch diese Aufgaben haben sie immer eine ausgeprägtere Dynamik als die Güterund Dienstleistungsmärkte.

\section{Kosten der Liberalisierung}

Es ist dennoch falsch, die generellen Eigenschaften moderner kapitalistischer Kreditgeldökonomien als Vorwand zu nehmen, die speziellen Entwicklungen auf den internationalen Finanzmärkten seit den siebziger Jahren als völlig unproblematisch zu sehen. Die Liberalisierung der internationalen Finanzmärkte verursacht Kosten, die sich in dreierlei Hinsicht bemerkbar machen.

Erstens geht die Liberalisierung der Finanzmärkte und der nationalen Zahlungsbilanztransaktionen mit einer signifikanten Zunahme von Finanz- und Währungskrisen einher. Empirische Studien belegen den Zusammenhang von Finanzmarktliberalisierung und dem Auftreten von Finanzkrisen. Ursachen der Krisen können die unzureichende institutionelle Struktur von Finanzmärkten (underbanking) sein, sie können mit 


\begin{tabular}{|c|c|c|}
\hline Gegenstand & Maßstab & Steigerungsfaktor \\
\hline 1. Bruttoinlandsprodukt & Faktorkosten (laufende Preise) & 1,5 \\
\hline 2. Investitionen & & 1,4 \\
\hline 3. Exporte & & 1,6 \\
\hline $\begin{array}{l}\text { 4. Direktinvestitionen } \\
\text { 5. (inward + outward) } \\
\text { 6. }\end{array}$ & $\begin{array}{l}\text { Stromzahlen } \\
\text { Bestände } \\
\text { Fusionen }\end{array}$ & $\begin{array}{l}5,5 \\
3,4 \\
7,6\end{array}$ \\
\hline 7. Aktien & $\begin{array}{l}\text { Marktkapitalisierung Jahresende } \\
\text { Umsätze }\end{array}$ & $\begin{array}{r}3,3 \\
10,0\end{array}$ \\
\hline 10. Anleihen & $\begin{array}{l}\text { Bestände } \\
\text { Umsätze }\end{array}$ & $\begin{array}{l}2,3 \\
7,1\left(^{\star}\right)\end{array}$ \\
\hline 11. Derivate & Bestände & $11,4(+)$ \\
\hline $\begin{array}{l}\text { 12. - börsengehandel } \\
\text { 13. }\end{array}$ & $\begin{array}{l}\text { Bestände } \\
\text { Umsätze (börslich) }\end{array}$ & $\begin{array}{l}6,2 \\
3,1\end{array}$ \\
\hline 14. - außerbörslich & Bestände & $14,9(+)$ \\
\hline
\end{tabular}

Quelle: Schlussbericht der Enquete-Kommission „Globalisierung der Weltwirtschaft - Herausforderungen und Antworten“. Bundestagsdrucksache 14/9200, Tabelle 2-1

dem $\mathrm{Zu}$ - und schnellem Abfluss kurzfristiger Finanztitel zusammenhängen oder auch, wie etwa bei der Asienkrise von 1997/98, das Ergebnis internationaler Kettenreaktionen sein. Die Folgen sind Vernichtung und/oder Unterauslastung von Produktionskapazitäten, die ganze Volkswirtschaften um Jahre in ihrer ökonomischen Entwicklung zurückwerfen können.

Zweitens hat im Zuge der beschleunigten ökonomischen Globalisierung die Volatilität weltwirtschaftlicher Preise stark zugenommen. In besonderer Weise gilt dies für die Wechselkurse, die seit dem Übergang zu einem Regime flexibler Kurse ausgeprägte Schwankungen aufweisen. Dies hat das Umfeld für international operierende ökonomische Akteure und deren Kalküle nachhaltig geändert. Exportorientierte Unternehmen entschieden sich angesichts der Unsicherheit über Wechselkurse verstärkt dazu, direkt in ausländischen Märkten zu produzieren, was zu einem Anstieg der ausländischen Direktinvestitionen

\section{Inserieren Sie in Ökologisches Wirtschaften!}

Sprechen Sie genau Ihre Zielgruppe an. Gesamtauflage von 1.500 Heften. Erscheint 5 x jührlich.

Fordern Sie noch heute die Mediadaten an!

Frau Katja Muchow berät Sie gerne.

\section{Kontakt:}

Katja Muchow c/ o INKA e.V.

Gravelottestr. 6, 81667 München

Fon 089/4591 19 19, Fax 089/4591 1920

E-Mail anzeigen@katja-muchow.de führte. Exporteure und Importeure von Waren und Dienstleistungen begannen sich gegenüber unliebsamen Wechselkursüberraschungen durch Zukunftstransaktionen abzusichern. Diese Hedging-Strategien ließen die internationalen Devisenmärkte anschwellen und trugen dazu bei, dass der Raum für spekulative Transaktionen zunahm. Neben dem Transaktionsvolumen ist vor allem die Transaktionshäufigkeit entsprechend stark gestiegen: Um eine grenzüberschreitende Waren- und/oder Dienstleistungstransaktion gegen Wechselkursschwankungen abzusichern, werden in der Regel vier bis fünf begleitende Devisenmarkttransaktionen fällig. Die immense Zahl täglicher Devisenmarkttransaktionen produzieren ein enormes Maß an noise, der wiederum die Grundlage für das zu beobachtende Herdenverhalten liefert: Wird der Informationsgehalt der Preise davon beeinträchtigt, kann bereits das Verhalten einiger weniger Marktmacher ausreichen, um panikartige Situationen zu erzeugen.

Drittens hat die Globalisierung der Finanzmärkte dazu beigetragen, dass sich der Zeithorizont ökonomischer Akteure verkürzt. Der short termism hat seine Gründe vor allem in den tiefgreifenden Änderungen der Finanzierungsumwelt ökonomischer Akteure. Die Internationalisierung der Finanzmärkte und die bereitgestellten neuen Finanzierungsinstrumente haben den nationalen Unternehmenssektoren auf der einen Seite größere und billigere Finanzierungsmöglichkeiten eröffnet und auf diese Weise, makroökonomisch gesehen, den Wachstumsprozess befördert. Auf der anderen Seite wurden die Unternehmen aber auch mit den Rentabilitätsansprüchen global agie- render Finanzintermediäre konfrontiert. Der Druck auf die Strukturen der corporate governance äußerte sich nicht nur in hohen Erwartungen an Ertragsraten, sondern vor allem in einer drastischen Verkürzung der Verwertungszeiträume. Aus dem Spektrum möglicher Investitionsprojekte werden unter diesen Bedingungen die ausgewählt, die in kürzester Frist die höchsten Profitraten erwarten lassen.

\section{- Nachhaltigkeit durch Zivilisierung der Finanzmärkte}

Diese drei Konsequenzen globalisierter Finanzmärkte bestimmen das Konfliktpotenzial für jede politisch in Gang gebrachte Strategie von Nachhaltigkeit. Viele nachhaltigkeitsorientierte Investitionen weisen höhere gesellschaftliche als private Ertragsraten und in den meisten Fällen einen längerfristigen Verwertungszeitraum auf. In diesen Fällen stellen die globalen Finanzmärkte ein Hindernis für einen nachhaltigen Pfadwechsel dar. Eine der wenigen empirischen Untersuchungen zu den Motiven "grüner" Finanzinvestoren zeigt die Reaktionen globalisierter Finanzmärkte auf die Entscheidungen ökologisch-ethisch motivierter Anleger (2): Obgleich diese Investorengruppe darauf achtet, dass ihre Finanzanlagen keine umweltschädlichen oder inhumanen Investitionen finanziert, gibt es doch eine Rentabilitätsschmerzgrenze für "grüne" Investitionen, die von den Ertragsraten der globalen Finanzmärkte vorgegeben wird. Nachhaltige Investitionen tun zweifelsohne Not, können aber erst dann zu einem nachhaltigen ökonomischen Faktor werden, wenn es gelingt, die globalisierten Finanzmärkte zu zivilisieren.

\section{Anmerkungen}

(1) Vgl. ausführlich Hübner, Kurt/ Petschow, Ulrich: Spiel mit Grenzen. Ökonomische Globalisierung und soziale Kohäsion, Berlin 2001.

(2) Lewis, Alan: A Focus Study of the Motivation to Invest: ,Ethical/green' and ,Ordinary' Investors compared, in: Journal of Socio-Economics, Vol. 30 (2001), S. 331-341.

\section{Der Autor}

Kurt Hübner ist Professor für internationale Wirtschafspolitik an der Fachhochschule für Wirtschaft Berlin sowie Gastprofessor am Canadian Centre for German and European Studies der York University in Toronto/ Kanada.

Kontakt: Fax 0041-6-736-5696,

E-Mail: khuebner@YorkU.ca 
(c) 20I0 Authors; licensee IÖW and oekom verlag. This is an article distributed under the terms of the Creative Commons Attribution Non-Commercial No Derivates License (http://creativecommons.org/licenses/by-nc-nd/3.o/), which permits unrestricted use, distribution, and reproduction in any medium, provided the original work is properly cited. 